DOI: $10.5007 / 2175-7941.2012 v 29 n e s p 2 p 679$

\title{
AMBIENTES DE MODELAGEM COMPUTACIONAL NO APRENDIZADO EXPLORATÓRIO DE FÍSICA
}

\author{
Laércio Ferracioli \\ Thieberson Gomes \\ Giuseppi Gava Camiletti
}

Laboratório de Tecnologias Interativas Aplicadas à Modelagem Cognitiva Departamento de Física - UFES

Vitória - ES

Rodrigo Marques Almeida da Silva

Doutorando Programa de Pós-Graduação de Informática - PUC-Rio

Rio de Janeiro - RJ

Mara Hombre Mulinari

Mestranda Programa de Pós-Graduação em Educação - UFES

EEEFM Sizenando Pechincha

Serra - ES

Rafael Rodrigues de Oliveira

Doutorando Programa de Pós-Graduação em Educação - UFES

EEEFM Antônio José Peixoto Miguel Nova Almeida

Serra - ES

Francis Carlos Morelato Marin

Escola Superior São Francisco de Assis

Santa Teresa - ES

Kathia Mariane Fehsenfeld

Faculdade Metropolitana de Rio do Sul

Rio do Sul - SC

Carlos Henrique Verbeno

Bolsista de Iniciação Científica ModeLab/FAPES/CNPq/UFES

Vitória - ES

\footnotetext{
${ }^{+}$Computer modelling environments into exploratory learning in Physics

* Recebido: fevereiro de 2012.

Aceito: junho de 2012.
} 


\title{
Resumo
}

Este trabalho apresenta, inicialmente, algumas visões sobre a interface tecnologia e ciência no contexto educacional, seguido do relato de três iniciativas independentes de investigação da utilização da modelagem computacional no ensino de ciências. Essas experiências, com o foco no conhecimento e não na tecnologia, são localizadas em um momento histórico em que o surpreendente avanço da tecnologia da informática passou a introduzir os computadores na vida cotidiana das pessoas e, consequentemente, levando-o para dentro da sala de aula. Na sequência, é apresentado um relato histórico do desenvolvimento e de resultados de um programa de pesquisa sobre integração de ambientes computacionais ao ensino de Física e ciências em geral a partir da modelagem computacional quantitativa, semiquantitativa e qualitativa no contexto educacional brasileiro.

Palavras-chave: Tecnologia \& ciência. Modelagem computacional. Aprendizado exploratório. Passos de construção de modelos.

\begin{abstract}
This paper presents, initially, different frameworks about the interface Technology and Science into the educational context, followed by the report of three independent experiences related to the study of the use of computer modelling in Science teaching. These experiences, focusing on knowledge, not on technology, happened in a specific moment when the striking advance of computer technology brought computers into classrooms. Subsequently, it is presented a report about the development of a research program about the infusion of computer environments in Physics teaching based on the use of quantitative, semi-quantitative and qualitative computer modelling environment into the Brazilian context.
\end{abstract}

Keywords: Technology \& science. Computer modelling. Exploratory learning. Steps for building models. 
... you should be suspicious of models, particularly if they are treated as true descriptions. Yet you should not despise models as childish. They pay an essential part in the human mind's method of perceiving and learning.

Eric Rogers, 1960

\section{Introdução}

A integração de ambientes computacionais em educação tem sido tema de investigação desde a década de 1970 e, em particular, com o foco na Física, podem-se citar Papert (1980), White \& Horwitz (1987) no estudo das Leis de Newton: a partir do conceito de micromundos, o primeiro explora as leis de movimento e o segundo explora atividades centradas na resolução de problemas e experimentação.

Uma perspectiva específica de investigação para a integração desses ambientes ao ensino de Física e Ciências, em geral, tem sido a modelagem computacional a partir do desenvolvimento de ambientes de modelagem computacional com características específicas. Alguns desses ambiente não se baseiam necessariamente em variáveis e suas relações matemáticas, mas em variáveis, relações de causa-efeito, objetos e eventos, tais como Ogborn (1984; 1987), Ogborn e Wong (1984), Ogborn e Holland (1986) e Bliss e Ogborn (1989) com amplos resultados relatados por Mellar et al. (1994).

Nesse contexto, este artigo relata a experiência do desenvolvimento de ambientes de modelagem computacional e investigação sobre sua utilização em diferentes contextos educacionais, iniciada em meados da década de 1990 pelo Grupo de Pesquisa em Ensino de Física da Universidade Federal do Espírito Santo (FERRACIOLI, 1995; 1997; 1999). A partir do ano de 2000, essa iniciativa passou a ser financiada pelo Conselho de Desenvolvimento Científico e Tecnológico através do Programa de Pesquisa Estudo da Integração de Ambientes Computacionais ao Aprendizado Exploratório em Ciências (FERRACIOLI, 2000).

\section{Perspectivas da tecnologia e ciência no contexto educacional}

Diferentemente dos dias de hoje, em que a tecnologia está massivamente presente em nossas vidas, embora ainda não tenhamos clareza sobre sua utilização na sala de aula, no final de década de 1980 e início da década de 1990, a busca de 
alternativas para a integração da temática tecnologia no contexto educacional gerou a publicação de diretrizes por setores educacionais de alguns países.

Nesse contexto, em 1996, o National Research Council norteamericano publica o National Science Education Standards, estabelecendo diretrizes para o ensino de ciência, articulando-o à temática tecnologia. O documento, talvez relembrando Eric Rogers (Rogers, 1960), adota um paradigma denominado ciência como investigação - science as inquiry -, definindo-o como um passo além de "ciência como um processo", que levaria o estudante a combinar processo e conhecimento científico, na medida em que utilizasse raciocínio científico e pensamento crítico no desenvolvimento de seu entendimento sobre ciência. Dessa forma, esse paradigma auxiliaria o estudante a desenvolver o entendimento de conceitos científicos; o entendimento da natureza da ciência; a valorização do "como sabemos" o que sabemos em ciências; as habilidades necessárias para tornar-se um questionador independente sobre o mundo que o rodeia; e a disposição para utilizar suas habilidades, competências e atitudes associadas à ciência (NRC, 1996, p. 105).

O documento, também, aborda a estreita relação entre os conceitos de ciência e tecnologia, distinguindo suas características a partir de seus objetivos: ciência tem o objetivo de entender o mundo que nos rodeia - natural world, enquanto tecnologia tem o objetivo de operar modificações no mundo ao nosso redor para atender a nossas necessidades específicas - designed/artificial world. Dessa forma, postula-se que ciência como investigação é o paralelo para

tecnologia como design com o foco no desenvolvimento de habilidades e entendimento dos limites, possibilidades e relações entre esses dois conceitos. Um importante aspecto é ressaltado quando aponta que "o uso de tecnologia não pode ser confundido com tecnologia instrucional que provê estudantes e professores com estimulantes ferramentas, tais como computadores, para desenvolver questionamentos e entender ciência" (NRC, 1996, p. 24).

Outro aspecto a ser ressaltado é que o documento inclui em suas diretrizes a formulação de explicações e construção de modelos, sejam estes físicos, conceituais ou matemáticos, para a diferenciação entre descrição e explicação, explicitando causas e seus efeitos, e estabelecendo relações baseadas em evidências e argumento lógico (NRC, 1996, p. 145, 175).

Em 1990, o Department of Education and Science inglês publica o Technology in the National Curriculum, estabelecendo diretrizes para o desenvolvimento de habilidades nessa temática, afirmando que tecnologia diz respeito a ações práticas baseadas no conhecimento e entendimento de um amplo espectro de tópicos e assuntos. A abordagem do tema é divida em duas vertentes: habilidades em design \& tecnologia e habilidades em tecnologia da informação. A primeira é 
relacionada à identificação de necessidades, geração e organização de ideias, planejamento e desenvolvimento de soluções práticas e testagem, na busca de melhores soluções; a segunda, relacionada à natureza e ao manuseio da informação e preparação para os avanços da tecnologia, sendo ressaltada a importância da transversalidade nas ações de sua implementação através de temas fundamentais ao longo de todo o currículo (DES, 1990, p. 4). O documento aponta que as habilidades nessa área deveriam incluir o desenvolvimento de ideias e a comunicação de informações; o manuseio de informações; a modelagem; a medição e o controle de dispositivos; e o entendimento dos limites de suas aplicações e de seus efeitos.

Em relação à modelagem, o documento afirma que modelos computacionais podem ser considerados representações do mundo que nos rodeia ou de situações abstratas abordando diferentes área de conhecimento. Os estudantes deveriam ser capazes de investigar, manipular e, ao final, construir seus próprios modelos abrangendo diferentes níveis de complexidade.

Em 1985, quando o Cometa Halley passou, a American Association for the Advancement of Science - AAAS - iniciou o Projeto 2061, visando promover o letramento em ciências, matemática e tecnologia de toda população, no entendimento de que um cidadão cientificamente letrado é ciente de que essas áreas representam um interdependente empreendimento humano com alcances e limitações, entendendo conceitos e princípios-chave da ciência, é familiarizado com o mundo natural que o rodeia, sendo capaz de identificar sua diversidade e unidade, e usa conhecimento e atitudes científicas para refletir sobre propósitos individuais e coletivos. O projeto aborda o conceito de tecnologia na visão de que, assim como a linguagem, os rituais, os valores, o comércio e as artes, a tecnologia é um elemento intrínseco do sistema cultural que delineia e espelha o sistema de valores vigentes, além de basear-se na ciência e, ao mesmo tempo, contribuir com esta, sendo usada na tentativa de adaptar o mundo às nossas necessidade (AAAS, 1990, p. 25).

Argumentando que algumas ideias transcendem os limites disciplinares, provando-se úteis aos processos de observação, explicação, construção de teoria e design, o projeto recomenda a utilização de temas, tais como sistemas e modelos. Um modelo é apontado como uma imitação simplificada de alguma coisa que acreditamos poder nos auxiliar a melhor entendê-la, podendo ser um dispositivo, um plano, um desenho, uma equação, um programa de computador ou mesmo uma imagem mental. Seja um modelo físico, matemático ou conceitual, o valor deles está em sua capacidade de nos fazer entender como as coisas funcionam ou deveriam funcionar. Quando um modelo não simula o fenômeno de forma adequada, eles podem induzir a erros mas, sendo analisados cuidadosamente, revelam característi- 
cas que não são apropriadas para o que está sendo modelado, sendo, então, um indicativo de que o modelo deve ser melhorado (AAAS, 1990, p. 168).

No Brasil, no final da década de 1990 e início da década de 2000, foram publicados os Parâmetros Curriculares Nacionais - PCN's - estabelecendo orientações para a adequação dos currículos à realidade contemporânea e aos avanços tecnológicos. Via de regra, esses documentos utilizavam a expressão ciência e tecnologia, não apresentando conceituação específica para cada um deles. Associavam a temática tecnologia à utilização de recursos tecnológicos ao afirmarem, por exemplo, que o uso do computador no ensino é particularmente importante nos dias de hoje ( $\mathrm{PCN}+, 2002 ;$ p 148), ou que deveria ser estimulado o uso adequado de meios tecnológicos, tais como máquinas de calcular ou ferramentas propiciadas pelos microcomputadores, especialmente editores de texto e planilhas $(\mathrm{PCN}+$, 2002, p. 112), sem, no entanto, apontar diretrizes específicas para essa adequação.

É importante ressaltar que alguns desses documentos, principalmente no contexto do Ensino de Ciências da Natureza e Matemática, orientam para a utilização de modelos, explorando o fato de que modelos em ciência são construídos a partir da necessidade explicativa de fatos, que podem ser tanto observados diretamente quanto inferidos, e que tais modelos são produtos da mente humana e não da própria natureza, construções mentais que procuram sempre manter a realidade observada como critério de legitimação (PCNEM, 2000, p. 25). Além desse aspecto, incluíam a orientação metodológica para uma perspectiva do ensino de ciências, em geral, e, em particular, para o ensino de Física e Química que utilizasse abordagens quantitativas e qualitativas orientando que o estudo deveria sempre ser iniciado pelos aspectos qualitativos e, só então, introduzir tratamento quantitativo (PCNEM III, 2000, p. 53).

\section{A modelagem computacional como alternativa de integração de tecnologia no contexto educacional}

As diretrizes descritas na seção anterior, de certa forma, construíram um cenário propício para o surgimento de uma pauta para a investigação do uso da modelagem computacional no contexto educacional, com o foco especial no ensino de física e ciência de maneira geral. Nesse contexto, são descritas três iniciativas correlatas. 


\section{III.1 Uma experiência inglesa}

Ogborn e colaboradores desenvolveram uma série de investigações sobre a utilização da ferramentas de modelagem computacional no contexto do ensino de ciências nas décadas de 1980 e 1990 (OGBORN, 1984; 1987; OGBORN; WONG, 1984; OGBORN; HOLLAND, 1986; BLISS; OGBORN, 1989; MELLAR at al., 1994). Para o contexto deste artigo, é importante relatar que o programa de pesquisa Tools for Exploratory Learning apresentado em Ogborn e Bliss (1989) descreve, entre outras, as seguintes questões de pesquisa:

- De que maneira a interação com ferramentas contendo representações nas área de tecnologia e ciências sociais do currículo pode facilitar a aprendizagem dessas áreas?

- Estudantes são auxiliados na aprendizagem quando representam e exploram as consequências de seus próprios modelos em um domínio?

- O que o estudante precisa saber e qual a idade apropriada para a utilização de ferramentas exploratórias e expressivas na construção de modelos?

- Como a utilização de ferramentas exploratórias e expressivas auxilia a construção de modelos?

- Como o estudante avalia um modelo construído por ele mesmo?

Resultados dessa investigação foram relatados em periódicos (e.g. BLISS et al., 1992; MILLER et al., 1993) e um abrangente relato poder ser encontrado em Mellar at al. (1994).

\section{III.2 Uma experiência suíça}

Vitale e colaboradores desenvolveram o projeto denominado L'Intégration de l'Informatique à La Pratique Pédagogique em uma perspectiva transdisciplinar, com a utilização de atividades de modelagem baseadas na linguagem LOGO (BÉGIN et al., 1994; 1995; 1996) Motivados pelo interesse em utilizar computadores em sala de aula de maneira inteligente, em oposição a uma postura passiva, e repensar a maneira que a "observação científica" era abordada no Ensino Fundamental, o projeto foi baseado na ideia de se utilizar a modelagem e a programação com os objetivos de:

- Criar um espaço transdisciplinar na escola;

- criar um referencial contextual e interpretativo para as atividades de laboratório de ciências;

- criar uma integração dinâmica da matemática e programação na educação em ciências. 
Além de publicações em congressos e periódicos (e.g. VITALE, 1991; 1993; 1995; 1998), um relato abrangente dos resultados dessa experiência pode ser encontrado em Vitale (1994).

\section{III.3 Uma experiência norteamericana}

Em 1993, um grupo de cientistas, professores de ciências, pesquisadores da área de educação, profissionais da área de ciências da computação e desenvolvedores de softwares, reuniram-se para o delineamento de um projeto de pesquisa partindo da premissa de que o foco na modelagem computacional e simulação como ferramentas para o entendimento de ciências e construção de novos conhecimentos tinha um potencial significativo para promover a melhora da qualidade do ensino de ciências e matemática no Ensino Fundamental e Médio. Muitos dos participantes traziam experiências anteriores nessa temática, e duas premissas foram apontadas: a primeira, que crianças deveriam ser engajadas no processo de construção, refinamento, utilização e validação de modelos de fenômenos naturais e sociais desde o início do processo de escolarização; a segunda, que atividades de modelagem computacional deveriam ter um papel central através do currículo escolar de ciências, não de modo periférico, nem de como parte especial ou opcional das disciplinas. $\mathrm{O}$ projeto foi orientado a partir dos seguintes questionamentos (NSF, 1996):

- Como poderiam atividades de modelagem ser introduzidas para estudantes de ciências de séries iniciais?

- Quais aspectos da cognição são afetados na aprendizagem através de um ambiente de modelagem?

- Que ferramentas de modelagem e aplicações são apropriadas?

- Como a visualização pode auxiliar a aprendizagem em ciências, a partir atividades de investigação baseadas na modelagem?

- Como a modelagem deveria ser integrada com observação, experimento e teoria?

- Como atividades colaborativas de modelagem promovem a melhoria da aprendizagem em ciências?

- Quais são as medidas apropriadas para a avaliação das habilidade de modelagem?

- Quais os impactos que a utilização da modelagem computacional para a igualdade social?

Algumas publicações significativas foram originadas dessa iniciativa, podendo-se citar, entre elas, a publicação de um número especial do periódico Inte- 
ractive Learning Environment com a temática Modeling and Simulation in Science Education no ano de 1994, que pode ser observado no endereço $<$ http://www.tandfonline.com/toc/nile20/4/3>, e a publicação de dois livros: o primeiro, relatando uma experiência de utilização do Ambiente de Modelagem Computacional Quantitativo STELLA em escolas de Ensino Médio (MANDINACH; CLINE, 1994) e o segundo, apresentando um amplo espectro de experiências com diferentes ambientes de modelagem computacional (FUERZEIG; ROBERTS, 1999).

O objetivo desta seção, em vez de apresentar resultados e repostas, foi o de apresentar questões levantadas por diferentes grupos de pesquisa e de interesse sobre a possibilidade de utilização da modelagem computacional nos processos de ensino e aprendizagem em Ciências, todas localizadas em um momento histórico em que o surpreendente avanço da tecnologia da informática passou a introduzir os computadores na vida cotidiana das pessoas e, consequentemente, levando-o para dentro da sala de aula.

Esse evento causou o surgimento de diferentes perspectivas de investigação, seja na área de Ensino de Ciências e Matemática, na área de Educação ou na área de Ciência da Computação, com o foco na tecnologia, com pouca ou nenhuma conexão com conteúdos curriculares ou transcurriculares específicos. No entanto, pode-se observar que o foco das experiências relatadas não era a tecnologia, mas o Ensino de Ciências de maneira geral; ou seja, o foco era, e sempre foi, o conhecimento, não a tecnologia (FERRACIOLI, 2011).

Nesse contexto, baseando-se em aspectos de diretrizes educacionais relacionadas à tecnologia e ao ensino de ciências, descritas na seção 2 , e em questões sobre a utilização da modelagem computacional no ensino de ciências descritas nessa seção, duas ações concomitantes foram tomadas no final da década de 1990 pelo Grupo de Pesquisa em Ensino de Física da Universidade Federal do Espírito Santo: a primeira foi a criação da disciplina optativa Informação, Ciência e Tecnologia no Ensino de Física na grade curricular da Graduação em Física (FERRACIOLI; SAMPAIO, 2001) e a segunda, o estabelecimento do Programa de Pesquisa Estudo da Integração de Ambientes Computacionais ao Aprendizado Exploratório em Ciências que, em 2000, passou a ser financiado pelo Conselho de Desenvolvimento Científico e Tecnológico (FERRACIOLI, 2000).

A criação da disciplina na graduação partiu do entendimento de que, ao contrário de várias iniciativas em universidades brasileiras, a tecnologia não deveria ser instituída como disciplina à parte, mas ser integrada como um potencial espaço a ser explorado e experienciado por professores das diversas áreas de conhecimento específico (FERRACIOLI, 1997). Nesse contexto, a proposta de traba- 
lho é de que a tecnologia da informática seja utilizada como ferramenta de conhecimento, como máquina capaz de ampliar a capacidade do estudante em formular perguntas e, muito menos, em simplesmente encontrar respostas (VITALE, 1995; FERRACIOLI, 1996).

O Programa de Pesquisa foi delineado com o foco na integração de ambientes de modelagem computacional aos processos de ensino e aprendizagem em Ciências a partir de três metas (FERRACIOLI, 2000):

- Estudo de diferentes ambientes de modelagem computacional através da proposição de atividades similares e paralelas;

- estudo do processo de construção/exploração de modelos nesses ambientes por parte dos estudantes;

- estudo do processo de modelagem computacional como estratégia de integração de tecnologia ao aprendizado exploratório em Ciências.

Investigar a integração de ambientes computacionais no aprendizado exploratório em ciências parte de relações entre ciência e tecnologia com o foco tanto na visão de ciência como investigação quanto na visão de tecnologia como design. Assim, busca-se o desenvolvimento de habilidades e entendimento dos limites, das possibilidades e das relações entre esses dois conceitos a partir da transversalidade nas ações de implementação através de temas básicos de todo o currículo. Dessa forma, a modelagem computacional é entendida como um elo para a construção dessa perspectiva de trabalho, uma vez que a natureza da modelagem propicia a exploração e/ou construção de mundos artificiais, podendo levar o estudante a engajar-se no processo de aprendizagem por ele mesmo, ou seja, no processo de aprendizagem exploratória em ciências.

Para finalizar, vale ressaltar que essas duas ações se retroalimentam sistemicamente na medida em que diretrizes traçadas no Programa de Pesquisa podem ser desenvolvidas no contexto da disciplina de graduação, e os resultados desta retroalimentam o programa.

\section{A modelagem computacional no aprendizado exploratório em ciên- cia $-1^{\text {a }}$ Etapa: Análise de ambientes de modelagem computacional}

A meta inicial desse programa foi o minucioso exame de ambientes de modelagem computacional enquadrados nas perspectivas quantitativa, semiquantitativa e qualitativa (BLISS et al., 1992), visando obter subsídios para o possível desenvolvimento de ambientes de modelagem melhor adaptados tanto para o desenvolvimento de pesquisa nesse campo de conhecimento quanto para sua integra- 
ção ao ensino da Física em diferentes níveis de escolaridade. Esse estudo foi desenvolvido a partir de atividades de modelagem exploratórias e expressivas: as primeiras quando o estudante é levado a explorar aspectos de um modelo desenvolvido anteriormente por um especialista no assunto abordado, e as segundas quando o estudante é solicitado a construir seu próprio modelo, com suas ideias e concepções e seus conceitos científicos (BLISS; OGBORN, 1989).

Para a estruturação das atividades de modelagem, foi estabelecida uma metodologia de trabalho denominada de Passos de Construção de Modelos (CAMILETTI, 2001), que consiste na:

$1^{\circ}$ Passo: Definição do sistema a ser estudado

$2^{\circ}$ Passo: Escolha do fenômeno de interesse no sistema escolhido

$3^{\circ}$ Passo: Listagem das variáveis relevantes

$4^{\circ}$ Passo: Construção do modelo no papel a partir de relações causais

$5^{\circ}$ Passo: Representação do modelo no ambiente de modelagem

$6^{\circ}$ Passo: Simulação do modelo construído

$7^{\circ}$ Passo: Validação do modelo a partir da análise de seu comportamento em relação ao comportamento esperado do fenômeno em estudo

Essa metodologia tem o objetivo de levar o estudante a refletir sobre o fenômeno abordado, preparando-o para a construção e representação de um modelo inicial no papel, para, então, ir ao computador representá-lo no ambiente de modelagem computacional: a representação informática é entendida como o estágio final da representação de um fenômeno (RAMPINELLI; FERRACIOLI, 2006). Essa metodologia produz a base de dados para a análise do processo de modelagem computacional.

A perspectiva quantitativa foi escolhida para o início do escrutínio dos ambientes de modelagem computacional. Ambientes de Modelagem Computacional Quantitativa, também denominados de ambientes de modelagem matemática, envolvem a especificação de variáveis relevantes de um sistema, seus valores e as relações algébricas entre elas. Inicialmente, foi escolhido o ambiente STELLA, acrônimo de Structural Thinking Experimental Learning Laboratory with Animation, cuja tradução livre para o Português é Laboratório de Aprendizagem Experimental com Animação baseado no Pensamento Sistêmico, seguido do Ambiente Vensim: ambos foram desenvolvidos baseados nos Princípios de Sistemas de Jay Forrester (FORRESTER, 1968), que procura entender o comportamento de um sistema a partir de relações causais entre as variáveis que o descreve. Esses ambientes são baseados na metáfora de taxas e níveis, através de uma interface gráfica amigável, que permite a análise dinâmica dos modelos criados. Uma funcionalida- 
de útil destas ferramentas é a construção, em segundo plano, das equações matemáticas representadas pelas relações estabelecidas no modelo. Devido ao fato do Ambiente STELLA ser um software pago, foi incorporada a análise do Ambiente Vensim, que disponibiliza uma versão grátis em seu portal, o que permitiu ampliar a amostragem dos estudos. Foram desenvolvidos estudos com amostras de estudantes de educação superior sobre Sistema Mola-Massa (CAMILETTI; FERRACIOLI, 2001); Estudo de Colisões em Laboratório (RAMPINELLI; FERRACIOLI, 2006); Leis de Newton (FEHSENFELD; FERRACIOLI, 2007); Métodos Numéricos de Integração (RABBI; FERRACIOLI, 2002) e estudantes de Ensino Médio sobre Cinemática de Translação (GONÇALVES, 2004; MARIN; FERREIRA; FERRACIOLI, 2007) e Resfriamento da Água (GONÇALVES; FERRACIOLI, 2011). Foram, ainda, desenvolvidos estudos em tópicos de Ciências relacionados ao Consumo de Água (DOMINICINI; CARNEIRO; FERRACIOLI, 2005); Ecossistemas Manguezais (MULINARI, 2006); e Crescimento Celular (MULINARI; FERRACIOLI, 2008). O Ambiente Modellus, onde os estudantes têm de produzir as equações que regem os fenômenos físicos abordados, foi também examinado em um Estudo da $2^{\mathrm{a}}$ Lei de Newton em Laboratório (VICTOR; FERRACIOLI, 2002). Além do exame desses ambientes, foi possível verificar que, apesar da natureza matemática do formalismo conceitual da Física que privilegiar um raciocínio quantitativo sobre o fenômeno abordado, esses ambientes demandam um aprendizado prévio para o sucesso do desenvolvimento das atividades propostas. A metodologia Passos de Construção de Modelos revelou-se apropriada para esse fim.

A perspectiva semiquantitativa foi a próxima a ser escrutinada. Ambientes de Modelagem Computacional Semiquantitativa envolvem a especificação de variáveis relevantes de um sistema, suas relações causais, mas analisa as tendências de variação dessas variáveis e não valores numéricos específicos, ou seja, não as quantifica. Bliss et al. (1992) apontam que estudos piagetianos sobre causalidade verificaram que, em várias situações do cotidiano, o raciocínio é baseado na direção, mas não o tamanho do efeito da ação de uma parte do sistema sobre outra. A ferramenta selecionada foi o Ambiente de Modelagem Computacional Semiquantitativa WLinkIt (SAMPAIO, 1996), que foi baseado no Ambiente IQON (KURTZ DOS SANTOS, 1992; KURTZ DOS SANTOS; OGBORN, 1994), para o qual foi desenvolvida a versão implementada denominada Ambiente VISQ (KURTZ DOS SANTOS; THIELO; KLEER, 1997). O Ambiente WLinkIt, disponível no endereço $<\underline{\mathrm{http}}$ ://www.nce.ufrj.br/ginape/wlinkit/>, é baseado na metáfora de ícones representados por caixas e links, através de uma interface gráfica de manipulação direta para a construção e simulação de modelos dinâmicos a partir de 
variáveis consideradas relevantes e sua relações causais. Foram desenvolvidos estudos com amostras de estudantes de educação superior sobre Sistema MolaMassa (CAMILETTI, 2001; CAMILETTI; FERRACIOLI, 2002a); Sistema Predador-Presa (CAMILETTI; FERRACIOLI, 2002b) e estudantes de Ensino Médio sobre Energia (OLIVEIRA, 2004). Esses estudos proveram os subsídios necessários para o desenvolvimento do Ambiente de Modelagem Computacional Semiquantitativa SQRLab, que será descrito na próxima seção.

Finalmente, a perspectiva qualitativa foi integrada ao programa de pesquisa. Ambientes de Modelagem Computacional Qualitativa permitem a construção de modelos sem o enfoque em variáveis, mas baseados em objetos e eventos que são gerados quando os objetos interagem entre si. Bliss et al. (1992) apontam que a construção de regras de interação requer, entre outras aspectos, o estabelecimento de um objetivo que implica na consideração de alternativas, avaliação de evidências, consideração de que se uma certa condição é imaginada então o que ocorrerá na sequência. A ferramenta selecionada foi o Ambiente de Modelagem Computacional Qualitativa WorldMaker (OGBORN; BOOHAN; WRIGHT, 1992; LAW; TAM, 1998), que permite a construção de modelos através da metáfora de objetos e eventos, consistindo na escolha dos objetos relevantes ao estudo de um sistema específico e das regras de interação que regem o comportamento destes objetos no sistema através de uma interface gráfica de manipulação direta. Assim, esse ambiente permite a construção de modelos que podem ser representados através de objetos que interagem entre si e com o meio e de eventos gerados por estas interações. Para o desenvolvimento dos estudos com esse ambiente, a metodologia Passos de Construção de Modelos utilizada nos estudos com ambientes quantitativos e semiquantitativos foi adaptada (GOMES, 2003) para:

$1^{\circ}$ Passo: Definição do sistema a ser estudado.

$2^{\circ}$ Passo: Escolha do fenômeno de interesse no sistema escolhido.

$3^{\circ}$ Passo: Listagem dos objetos relevantes.

$4^{\circ}$ Passo: Classificação dos objetos listados em Atores e Cenário.

$5^{\circ}$ Passo: Listagem em papel das regras de interação entre os objetos na estrutura se ... então.

$6^{\circ}$ Passo: Criação das regras de interação no papel de acordo com a estrutura de criação de regras do ambiente de modelagem.

$7^{\circ}$ Passo: Implementação das regras de interação no ambiente de modelagem.

$8^{\circ}$ Passo: Simulação do modelo construído.

$9^{\circ}$ Passo: Validação do modelo a partir da análise de seu comportamento em relação ao comportamento esperado do fenômeno em estudo. 
A partir dessa metodologia, foram desenvolvidos estudos com estudantesingressantes de educação superior sobre Difusão de Gases (GOMES, 2003; GOMES; FERRACIOLI, 2006a; OLIVIERA, 2006) e Sistema Predador-Presa (GOMES; FERRACIOLI, 2005; GOMES; FERRACIOLI, 2006b). Esses estudos proveram os subsídios necessários para o desenvolvimento do Ambiente de Modelagem Computacional Qualitativa ModeLab², que será descrito na próxima seção.

\section{A modelagem computacional no aprendizado exploratório em ciên- cia $-2^{\mathrm{a}}$ Etapa: $\mathrm{O}$ desenvolvimento de ambientes de modelagem compu- tacional}

A partir dos estudos até aqui relatados, foi possível escrutinar as potencialidades e limitações das ferramentas examinadas para o desenvolvimento da pesquisa sobre a modelagem computacional, tanto no contexto da ciência e tecnologia, com o foco no conhecimento, quanto para sua integração ao ensino da Física em diferentes níveis de escolaridade, baseada na metodologia desenvolvida no percurso desse programa de pesquisa. Dessa forma, a etapa seguinte foi o desenvolvimentos de um ambiente de modelagem computacional qualitativa e um ambiente de modelagem computacional semiquantitativa.

V.1 O ambiente de modelagem computacional qualitativa ModeLab 2D ou ModeLab ${ }^{2}$

O Ambiente de Modelagem Computacional Qualitativa denominado ModeLab ${ }^{2}$, acrônimo de Modelling Laboratory 2D, está disponível no endereço $<$ modelab2.modelab.org $>$, juntamente com uma versão do Manual do Usuário e foi desenvolvido com financiamento do $\mathrm{CNPq}^{1}$. O estudo da modelagem de sistemas com o Ambiente WorldMaker permitiu a construção de um ambiente com novo design gráfico para as funcionalidades e a inclusão de ferramentas de coleta de dados direcionadas à investigação do processo de modelagem, tais como Ferramenta de Exportação da Estrutura do Modelo, que auxilia e otimiza a análise dos modelos construídos pelos estudantes; Gerador de Arquivo Log, que registra todos os procedimentos dos estudantes durante a atividade de construção de modelos; e Estrutura de Regras, que possibilita estabelecer as conexões entre os objetos

\footnotetext{
1 A integração da modelagem computacional baseada nas regras de autômatos celulares no aprendizado exploratório em ciências, Contratos n. 403257/2003-4, 477028/2006-3, 503157/2004-0 e 505663/2003-1.
} 
em relação a suas regras através da linguagem visual de um grafo. Até o momento, foram desenvolvidos estudos sobre Expansão de Gases e Sistema Predador-Presa (GOMES, 2008); Velocidade Média (POLONINE; GOMES; FERRACIOLI, 2009); Cinética dos Gases (FEHSENFELD; FERRACIOLI, 2009a); Sistemas Complexos (FEHSENFELD; FERRACIOLI, 2009b); Energia Térmica e Gás Confinado em um Recipiente (FEHSENFELD, 2010). Foi, também, desenvolvido um estudo sobre Ciclo Hidrológico (BIASUTTI, 2009), além de uma investigação sobre utilização deste ambiente no estudo de Cinemática de Translação com estudantes de Ensino Médio que está em andamento (OLIVEIRA; FERRACIOLI, 2011; 2012).

Esses estudos revelaram resultados relativos ao Ambiente ModeLab ${ }^{2}$ propriamente dito e ao desempenho de estudantes no desenvolvimento de atividades de modelagem com o mesmo. Os primeiros mostraram que a implementação das ferramentas de coleta de dados direcionadas à investigação do processo de modelagem desempenharam um papel preponderante para o processo de análise de dados, reduzindo consideravelmente seu tempo e, consequentemente, apontando para a viabilidade do delineamento de estudos com amostras maiores, na busca de maior sustentação empírica para os resultados encontrados, fator crucial para a pesquisa nessa área. Em relação à utilização do ambiente pelos estudantes, nos casos de atividades de modelagem expressivas, os resultados revelaram que todos foram capazes de construir modelos sobre os sistemas propostos e estes apresentaram, para a maioria dos casos, o comportamento esperado.

Nesse contexto, a relevância dos Passos de Construção de Modelos é determinante, uma vez que o estudante é introduzido ao processo de modelagem através da construção de modelos no papel, seguido de sua representação no Ambiente de Modelagem, levando-o a refletir quanto à visão geral do sistema a ser representado e quanto ao processo de construção do modelo desse sistema. Nos estudos baseados em atividades exploratórias (POLONINE; GOMES; FERRACIOLI, 2009; BIASUTTI, 2009; OLIVEIRA; FERRACIOLI, 2011; 2012), os resultados apontaram que, devido à natureza aleatória da simulação no Ambiente $\mathrm{ModeLab}^{2}$, foi possível desenvolver atividades com o foco na experimentação, através do quais o estudante era levado a simular a mesma situação por um numero de vezes e, ao final, ser capaz de desenvolver habilidades relacionadas ao entendimento de conceitos abordados, tais como impacto ambiental urbano, valor médio, velocidade média. Nesse contexto, a relevância do design gráfico do Ambiente ModeLab ${ }^{2}$ tem sido um fator determinante, uma vez que a interação estudanteambiente não tem se constituído em dificuldade para o desenvolvimento das atividades de modelagem propostas. 


\section{V.2 O ambiente de modelagem computacional semiquantitativa SQRLab}

O Ambiente de Modelagem Semiquantitativa denominado SQRLab, acrônimo de Semiquantitative Reasoning Laboratory, está disponível no endereço <sqrlab.modelab.org>, juntamente com uma versão do Manual do Usuário, e foi desenvolvido com financiamento do $\mathrm{CNPq}^{2}$.

$\mathrm{O}$ estudo da modelagem de sistemas com o Ambiente WLinkIt permitiu a construção de um ambiente com design gráfico similar ao do Ambiente ModeLab ${ }^{2}$ e à inclusão de ferramentas de coleta de dados direcionadas para a investigação do processo de modelagem, tais como, Editor de Propriedades e Propriedades, que são caixas de diálogo laterais de acesso rápido a todas a variáveis e ligações que compõem o modelo construído pelo estudante e que auxiliam e otimizam a sua análise; Gerador de Arquivo Log, registra todos os procedimentos dos estudantes durante a atividade de construção de modelos; Janela de Gráficos, possibilitando a visualização de saídas gráficas relativas a sucessivas simulações, possibilitando a verificação visual de qualquer alteração no modelo em estudo; e Gerador de Diagramas Causais de um modelo construído no formato de um arquivo de imagem. Essa característica foi trazida do Ambiente de Modelagem Computacional Quantitativo STELLA.

Até o momento, foram desenvolvidos estudos sobre Aquecimento Global com estudantes de Ensino Médio (MARIN, 2009) e um estudo sobre Sistemas Oscilantes com estudantes de educação superior está em andamento (VERBENO et al., 2011).

O primeiro estudo, desenvolvido como atividade extra-classe e baseado nos Passos de Construção de Modelos, revelou que, apesar de dificuldades iniciais relacionadas às definições de sistema e fenômeno de interesse, os estudantes adquiriram habilidade para a construção de diagramas causais e sua representação no Ambiente SQRLab, que se mostrou adequado como recurso de construção e simulação de modelos, uma vez que auxiliou os estudantes tanto na validação do modelo construído quanto na verificação da previsão de comportamento gráfico construídos no papel.

No segundo estudo, foi possível observar que os estudantes foram levados a refletir sobre suas próprias concepções sobre sistemas oscilantes e os resultados das atividades de modelagem revelaram indícios de uma evolução conceitual em direção ao conhecimento científico sobre o tema.

\footnotetext{
${ }^{2}$ Integração da modelagem computacional no aprendizado exploratório em ciências, Contratos n. 477028/2006-3.
} 


\section{V.3 Um ambiente de modelagem computacional quantitativa}

A experiência adquirida nos estudos de ambientes de modelagem computacionais relatados e o desenvolvimento e estudo do processo de modelagem com os Ambientes ModeLab ${ }^{2}$ e SQRLab através da metodologia de trabalho baseada nos Passos de Construção de Modelos com o foco no ensino da Física apontaram para a necessidade de desenvolvimento de uma ferramenta quantitativa pela própria natureza dessa área de conhecimento. Dessa forma, uma versão quantitativa com interface gráfica seguindo o mesmo design do Ambiente SQRLab que, por sua vez, segue, em linhas gerais, o design gráfico do Ambiente ModeLab ${ }^{2}$, está em fase final de testagem para ser disponibilizado.

\section{A modelagem computacional no aprendizado exploratório em ciên- cia - Próxima etapa: Perspectivas do futuro}

O estabelecimento da disciplina optativa Informação, Ciência e Tecnologia no Ensino de Física na grade curricular da Graduação em Física tem propiciado o desenvolvimento de alguns dos estudos aqui relatados. A disciplina é organizada no formato de desenvolvimento de projetos ao longo do semestre, levando os estudantes a caminharem por todo o processo de elaboração, desenvolvimento com coleta de dados e comunicação de resultados.

Nesse contexto, uma das temáticas de projetos é a utilização da modelagem computacional para o ensino da Física, o que tem gerado resultados de destaque pelos estudantes que, ao concluírem o projeto, são incentivados a escrever um artigo para submetê-lo a algum congresso na área de Ensino de Física. Exemplares dessa situação são o Estudo da $1^{\text {a }}$ Lei da Termodinâmica (MOTA et al., 2005) e Estudo sobre os Movimentos Uniforme e Uniformemente Variado (MARIN; FERREIRA; FERRACIOLI, 2007) e Leis de Newton (FEHSENFELD; FERRACIOLI, 2007).

A disciplina já foi ofertada para o Curso de Ciências Biológicas, gerando resultados similares (e.g. MULINARI; FERRACIOLI, 2008; DOMINICINI; CARNEIRO; FERRACIOLI, 2005), e tem se revelado como uma perspectiva promissora pelos resultados obtidos à medida que a temática ciência, tecnologia e inovação se faz cada vez mais presente no cotidiano escolar. Um fato que corrobora essa perspectiva é a inclusão de disciplina similar na Licenciatura em Física EaD da Universidade Federal do Espírito Santo para a qual foi produzida a publicação Informação, Tecnologia e Ciência no Ensino de Física (CAMILETTI et al. 2011). 
Outra perspectiva que se descortina para um futuro de médio-longo prazo é o Programa de Pós-Graduação em Ensino de Física - Mestrado Profissional da Universidade Federal do Espírito Santo, estabelecido em 2011 e voltado para Professores de Física, em que as dissertações devem gerar produtos associados à abordagem de situações-problema do cotidiano de sala de aula. Considerando que a modelagem computacional no ensino de Física é o foco de uma das linhas de pesquisa do Mestrado e o fato de que a investigação dos ambientes de modelagem qualitativa, semiquantitativa e quantitativa, descritos acima, têm gerado resultados promissores, há uma perspectiva concreta de aprofundamento tanto estudo dos processos de construção/exploração de modelos nesses ambientes por parte dos estudantes, quanto do estudo da modelagem computacional como estratégia de integração de tecnologia ao aprendizado exploratório em Física, metas do Programa de Pesquisa que gerou o desenvolvimento desses ambientes (FERRACIOLI, 2000). Pode-se, ainda, conjugar o paradigma modelagem-simulação-visualização (FUERZEIG; ROBERTS, 1999), explorando a visualização científica a partir da observação da evolução temporal do sistema modelado diretamente na área de visualização, ou através das saídas gráficas disponíveis.

O desenvolvimento dos ambientes de modelagem computacional, com interface gráfica similares e de manipulação direta, teve o objetivo de reduzir a inevitável carga cognitiva relacionada ao aprendizado de ferramentas computacionais, permitindo que o estudante concentre-se na tarefa de conteúdo específico a ser desenvolvida (ROCHA; BARANAUSKAS, 2003). Uma vez disponibilizado o ambiente quantitativo, será possível aprofundar a meta de estudar o processo de construção/exploração de modelos nesses ambientes por parte dos estudantes, e investigar a hipótese de trabalho de que o pensamento qualitativo é precursor do pensamento semiquantitativo, que, por sua vez, é precursor do pensamento quantitativo, estágio apropriado para o tratamento e formulação de conceitos da Física (KURTZ DOS SANTOS; SAMPAIO; FERRACIOLI, 2000).

\section{Considerações finais}

Este trabalho apresentou um relato do desenvolvimento do programa de pesquisa Estudo da Integração de Ambientes Computacionais ao Aprendizado Exploratório em Ciências, que investiga a modelagem computacional em suas perspectivas qualitativa, semiquantitativa e quantitativa, apresentando resultados para o contexto do ensino da Física. Inicialmente, foram apresentadas visões oficiais sobre a interface tecnologia e ciência no contexto educacional, descrevendo três iniciativas independentes de investigação da utilização da modelagem compu- 
tacional no ensino de ciências que, de certa forma, espelham as visões oficiais, dado que o foco das iniciativas é no conhecimento e não na tecnologia.

Na medida em que, nos dias de hoje, a tecnologia torna-se ubíqua, ela simplesmente é ou está. Assim, passa a não ter sentido falar em trazer a tecnologia para a sala de aula: ela já está na sala de aula com cada estudante com seu tablet, smartphone, ou qualquer engenhoca de última geração.

Nessa perspectiva, parece-nos que a clássica tríade professor-alunomaterial instrucional também perde o sentido, quando a tecnologia é associada a material instrucional, uma vez que ela perpassa ou impregna todas as dimensões de nossas vidas, incluindo a vida escolar: a tecnologia está tornando-se invisível (NORMAN, 1998), daí a necessidade do foco no conhecimento em qualquer proposta de ensino mediada por tecnologias.

Assim, a modelagem, seja ela de papel e lápis, física, matemática ou computacional, pode representar uma alternativa concreta para o ensino da Física. $\mathrm{Na}$ visão de ciência como investigação, que busca o entendimento do mundo que nos rodeia, a modelagem mimetiza os passos desse processo na medida em que promove (NRC, 1996; NSF, 1996):

- Observação;

- Levantamento de hipóteses;

- Proposição de questões;

- Análise de fontes de informação na busca do que já é conhecido;

- Revisão do que já é conhecido à luz de evidências experimentais;

- Planejamento e condução de experimentos;

- Utilização de ferramentas e técnicas adequadas para coletar, analisar e interpretar dados;

- Utilização do pensamento crítico e lógico entre evidências e proposição de respostas, explicações e predições;

- Consideração de explicações alternativas;

- Comunicação de resultados.

$\mathrm{Na}$ abordagem da modelagem computacional, o paradigma é tecnologia como design, através do qual pode-se levar o estudante a construir um mundo artificial e operar modificações para atender a necessidades e demandas específicas ao ensino, por exemplo, levando-o a comparar resultados gerados pela simulação do modelo com resultados obtidos em laboratório, ou construir um modelo a partir de dados obtidos em laboratório, ou, ainda, na análise de séries pretéritas de dados para a previsão de cenários futuros (DS, 1990; 1991; VITALE, 1993). 
Os resultados relatados neste artigo apontam que a integração de ambientes computacionais ao ensino da Física a partir da modelagem computacional quantitativa, semiquantitativa e qualitativa vem se revelando uma estratégia promissora. Nesse contexto, o foco não é na tecnologia, mas sim em conteúdos curriculares específicos estruturados em Módulos Educacionais e baseados nos Passos de Construção de Modelos. Nessa perspectiva, o estudante é levado a refletir sobre o seu conhecimento prévio em relação ao tema abordado, sobre suas concepções que dão suporte a esse seu conhecimento e sobre os conceitos científicos em estudo para promover sua evolução conceitual, levando-o a articular processo e conhecimento na medida em que utiliza o raciocínio científico e o pensamento crítico na construção de seu entendimento sobre a Física.

\section{Agradecimento}

Trabalho parcialmente financiado pelo CNPq, CAPES, FACITEC/PMV Fundo de Apoio à Ciência e Tecnologia do Conselho Municipal de Ciência e Tecnologia do Município de Vitória, ES e FAPES-ES.

\section{Referências bibliográficas}

AAAS (American Association for the Advancement of Science). Science for All Americans: Project 2061. New York: Oxford University Press. 1990.

BÉGIN, C.; GURTNER, J. L.; MARCELLUS, O.; DENZLER, M.; TRYPHON, A.; VITALE, B. Activités de représentation et de modélisation dans une approche exploratoire de la mathématique et des sciences. Part I: Les activités de représentation. Petit X, n. 38, p. 41-71, 1994

BÉGIN, C.; GURTNER, J. L.; MARCELLUS, O.; DENZLER, M.; TRYPHON, A.; VITALE, B. Activités de représentation et de modélisation dans une approche exploratoire de la mathématique et des sciences. Part II: Les activités de modélisation dans le continu. Petit X, n. 41, p. 51-82, 1995.

BÉGIN, C.; GURTNER, J. L.; MARCELLUS, O.; DENZLER, M.; TRYPHON, A.; VITALE, B. Activités de représentation et de modélisation dans une approche exploratoire de la mathématique et des sciences. Part III: Les activités de modélisation dans le discret. Petit X, n. 42, p. 5-27, 1996. 
BIASUTTI, L. D. Utilização da modelagem computacional qualitativa para o estudo do fenômeno do ciclo hidrológico. 2009. 99 f. Trabalho de Conclusão de Curso. (Graduação em Ciências Biológicas) - UFES, Vitória, ES.

BLISS, J.; OGBORN, J. Tools for Exploratory Learning. Journal od Computer Assisted Learning, Bristol, UK, v. 5, n. 1, p. 37-50, 1989.

BLISS, J.; OGBORN, J.; BOOHAN, R.; BRIGGS, J.; BROSNAN, T.; BROUGH, D.; MELlAR, H.; MILlER, R.; NASH, C.; RODGERS, C.; SAKANIDIS, B. Reasoning Supported by Computacional Tools. Computers in Education, UK, v. 18, n. 1, p. 1-9, 1992.

CAMILETTI, G. G.; FERRACIOLI, L. A utilização da modelagem computacional quantitativa no aprendizado exploratório de Física. Caderno Catarinense de Ensino de Física, Florianópolis, v. 18, n. 2, p. 214-228, 2001.

CAMILETTI, G. G.; FERRACIOLI, L. A utilização da modelagem computacional semiquantitativa no estudo do sistema massa-mola. Revista Brasileira de Ensino de Física, São Paulo, v. 24, n. 2, p. 110-123, 2002a.

CAMILETTI, G. G.; FERRACIOLI, L. The use of semiquantitative computer modelling in Science Education: the study of predator-prey system. In: INTERNATIONAL ORGANIZATION FOR SCIENCE AND TECHNOLOGY EDUCATION SYMPOSIUM, 10, 2002, Foz do Iguaçu, Brasil. Proceedings ... São Paulo, 2002b. p. 260-268.

CAMILETTI, G. G.; A modelagem computacional semiquantitativa no estudo de tópicos de ciências: um estudo com estudantes universitários. $2001.218 \mathrm{f}$. Dissertação (Mestrado em Física) - Centro de Ciências Exatas, UFES, Vitória, ES.

CAMILETTI, G. G.; GOMES, T.; FERREIRA, L.B.; FERRACIOLI, L. Informação, tecnologia \& ciência no ensino de Física. Vitória, ES: GSA Editora, 2011.

DES (DEPARTMENT OF EDUCATION \& SCIENCE). Science in the National Curriculum. London, UK: HMSO Publication Centre. 1991.

DES (DEPARTMENT OF EDUCATION \& SCIENCE). Technology in the National Curriculum. London, UK: HMSO Publication Centre. 1990.

DOMINICINI, C. K.; CARREIRO, F. da E; FERRACIOLI, L. O estudo do consumo de água através da modelagem computacional quantitativa. In: ENCONTRO 
NACIONAL DE ENSINO DE BIOLOGIA, I, 2005, Rio de Janeiro. Anais... 2005. p. 592-598.

FEHSENFELD, K.M.; FERRACIOLI, L. Desenvolvimento, utilização e avaliação de um módulo educacional baseado na modelagem computacional no contexto da sala de aula: um estudo sobre a primeira e a segunda leis de Newton. In: ENCONTRO NACIONAL DE PESQUISA EM EDUCAÇÃO EM CIÊNCIA, 6, 2007, Florianópolis, SC. Disponível em: <http://www.fae.ufmg.br/abrapec/viempec/viempec/> Acesso em: 24 jan. 2012.

FEHSENFELD, K.M.; FERRACIOLI, L. A definição de objetos e eventos na modelagem de fenômenos relacionados à cinética dos gases utilizando o ambiente de modelagem qualitativa ModeLab2. In: SIMPÓSIO NACIONAL DE ENSINO DE FÍSICA, 18, 2009, Vitória, ES. 2009a. Disponível em:

$<$ http://www.sbf1.sbfisica.org.br/eventos/snef/xviii/> Acesso em: 24 jan. 2012.

FEHSENFELD, K.M.; FERRACIOLI, L. Using ModeLab ${ }^{2}$ in complex systems modelling. In: ENCONTRO NACIONAL DE FÍSICA DA MATÉRIA CONDENSADA, 32, 2009, Águas de Lindóia, SP. Abstracts... 2009b. p. 124.

FEHSENFELD, K.M. A representação de fenômenos de cinética de gases utilizando o ambiente de modelagem computacional qualitativa ModeLab ${ }^{2}$ : um estudo exploratório com estudantes ingressantes na Educação Superior. 2010. Tese (Doutorado em Física) - Centro de Ciências Exatas, UFES, Vitória, ES.

FERRACIOLI, L. Novas tecnologias: a informática no ensino de Física. In: SIMPÓSIO NACIONAL DE ENSINO DE FÍSICA, 11, 1995, Niterói. Atas... Niterói: UFF, 1996. p. 39-45.

FERRACIOLI, L. As novas tecnologias nos centros de ciências, nos centros de formação profissional e na formação de professores. In: SIMPÓSIO NACIONAL DE ENSINO DE FÍSICA, 12, 1997, Belo Horizonte. Atas... Belo Horizonte, Imprensa Universitária da UFMG, 1997. p. 127-131.

FERRACIOLI, L. (1999) A Modelagem do Raciocínio sobre a Reversibilidade de Processos. In: SEMINÁRIO SOBRE MODELAGEM NO PROCESSO DE ENSINO-APRENDIZAGEM, 3, 1999, Rio Grande. Atas ... Rio Grande: Editora da FURG, 1999. p. 66-79. 
FERRACIOLI, L. Estudo da integração de ambientes computacionais ao aprendizado exploratório em ciências. Projeto de Pesquisa financiado pelo CNPq. Contrato 46.8522/00-0. 2000.

FERRACIOLI, L. Perspectivas da Modelagem em Educação em Ciências e Tecnologia para a Formação de Professores através do Ensino à Distância. (Org.). In: SEMINÁRIO SOBRE REPRESENTAÇÕES E MODELAGEM NO PROCESSO DE ENSINO-APRENDIZAGEM, IV, 2003, Vitória. Anais... Vitória, ES: Gráfica e Editora Mabor. Disponível em: $<$ www.modelab.ufes.br/ivseminario $>$.

FERRACIOLI, L. O conhecimento é o foco, não a tecnologia: a busca de caminhos entre educação em ciência e tecnologia e diversidade e práticas educacionais inclusivas. In: SEMINÁRIO DIVERSIDADE E PRÁTICAS EDUCACIONAIS INCLUSIVAS, 1, 2011, Vitória. Anais... Vitória, ES: PPGE/UFES, 2011.

FERRACIOLI, L.; SAMPAIO, F. F. Informação, ciência, tecnologia \& inovação curricular em Cursos de Licenciatura. Revista Brasileira de Informática na Educação, Florianópolis, v. 8, n. 2, p. 83-88, 2001.

FEURZEIG, W.; ROBERTS, N. (Eds.) Modelling and simulation in Science and Mathematics Education. New York: Springer-Verlag, 1999.

FORRESTER, J. Principles of systems. Cambridge: Wright-Allen Press, 1968.

GOMES, T. A modelagem computacional qualitativa no estudo de tópicos de ciências: um estudo exploratório com estudantes universitários. 2003. 285 f. Dissertação (Mestrado em Física) - Centro de Ciências Exatas, UFES, Vitória, ES.

GOMES, T. A modelagem computacional qualitativa através do ambiente ModeLab ${ }^{2}$ : um estudo exploratório com estudantes universitários desenvolvendo atividades de modelagem expressiva sobre tópicos de ciências. 2008. 271 f. Tese (Doutorado em Física) - Centro de Ciências Exatas, UFES, Vitória, ES.

GOMES, T.; FERRACIOLI, L. A utilização da modelagem computacional qualitativa no estudo do sistema predador-presa. Revista Brasileira de Informática na Educação, Porto Alegre, v. 13, n. 3, p. 51-59, 2005. 
GOMES, T.; FERRACIOLI, L. A investigação da construção de modelos no estudo de um tópico de Física utilizando um ambiente de modelagem computacional qualitativo. Revista Brasileira de Ensino de Física, v. 28, n. 4, p. 1-9, 2006a.

GOMES, T.; FERRACIOLI, L. The use of qualitative computer modelling environment in Science Education: a study with the predator-prey system. In: JANIUK, R. M.; SAMONEK-MICIUK, E. (Org.). Science and technology education for a diverse world: dilemmas, needs and partnership. 1. ed. Lublin, Polônia: Marie Curie-Sklodowska University Press, 2006b. v. 1. p. 239-251.

GONÇALVES, E. Um estudo da modelagem computacional quantitativa através de estruturas causais básicas: um estudo exploratório com estudantes do Ensino Médio Tecnológico. 2004. 181 f. Dissertação (Mestrado em Física) Centro de Ciências Exatas, UFES, Vitória, ES.

KURTZ DOS SANTOS, A. C.; OGBORN, J. Sixth form students' ability to engage in computational modelling. Journal of Computer Assisted Learning, Bristol, UK, v. 10, n. 3, p. 182-200, 1994.

KURTZ DOS SANTOS, A. C.; THIElO, M. R.; KLEER, A. A. Students modelling environmental issues. Journal of Computer Assisted Learning, London, v. 13, n. 1, p. 35-47, 1997.

KURTZ DOS SANTOS, A. C. Computational modelling in Science Education: a study of students' ability to manage some different approaches to modelling. 1992. Tese (Doutorado em Educação) - Institute of Education University of London, London, UK.

KURTZ DOS SANTOS, A. C.; SAMPAIO, F. F; FERRACIOLI, L. Um experimento de modelagem dinâmica semiquantitativa com a utilização da técnica dos hexágonos. Revista Brasileira de Informática na Educação, Florianópolis, v. 7, n. 3, p. 21-35, 2000.

LAW, N.; TAM, E. W. C. WorldMaker(HK): an iconic modelling tool for children to explore complex behaviour. In: INTERNATIONAL CONFERENCE ON COMPUTERS IN EDUCATION, 6, 1998, China. Proceedings...

MANDINACH E. B.; CLINE, H. Classrooms dynamics: implementing technology-based learning environment. New Jersey: Lawrence Erlbaum, 1994. 
MARIN, F. C. M.; FEREIRA, T. Q. S.; FERRACIOLI, L. Desenvolvimento e avaliação de um módulo educacional baseado na modelagem computacional: relato de um projeto na disciplina Informação, Ciência \& Tecnologia no Ensino de Ciências. In: SIMPÓSIO NACIONAL DE ENSINO DE FÍSICA, 17, 2007, São Luiz, MA. Disponível em: <http://www.sbfl.sbfisica.org.br/eventos/snef/xvii/atas/>. Acesso em: 24 jan. 2012.

MARIN, F. C. M. A utilização de diagramas causais no desenvolvimento de atividades de modelagem semiquantitativa: um estudo exploratório com estudantes do Ensino Médio. 2009. 325 f. Dissertação (Mestrado em Física) - Centro de Ciências Exatas, UFES, Vitória, ES.

MEC/SEF (1998) Parâmetros Curriculares Nacionais: introdução aos PCN's. Brasília: MEC/SEF.

MEC/SEMTEC (2002) PCN + Ensino Médio: ciências da natureza, matemática e suas tecnologias. Brasília: MEC/SEMTEC.

MELLAR, H.; BLISS, J.; BOOHAN, R.; OGBORN, J.; TOMPSETT, C. Learning with artificial worlds: computer based modelling in the curriculum. London: The Falmer Press, 1994. 244 p.

MILLER, R.; OGBORN, J.; BRIGGS, J.; BROUGH, D.; BLISS, J.; BOOHAN, R.; BROSNAN, T.; MELLAR, H.; SAKANIDIS, B. educational tools for computacional modelling. Computers in Education, UK, v. 21, n. 3, p. 205-261, 1993.

MOTA, R. de O.; COMPOSTRINI, L.; RAPINELLI, M.; FERRACIOLI, L. Desenvolvimento e avaliação de um módulo educacional sobre termodinâmica baseado na modelagem computacional quantitativa. In: SIMPÓSIO NACIONAL DE ENSINO DE FÍSICA, 16, 2005, Rio de Janeiro. Disponível em:

$<$ http://www.sbfl.sbfisica.org.br/eventos/snef/xvi/cd/index.html $>$ Acesso em: 24 jan. 2012.

MULINARI, M. H. A utilização da modelagem computacional como estratégia de análise de ecossistemas manguezais. 2006. 56 f. Trabalho de Conclusão de Curso. (Graduação em Ciências Biológicas) - UFES, Vitória, ES.

MULINARI, M. H.; FERRACIOLI, L. A utilização da tecnologia da informação no ensino de biologia: um experimento com um ambiente de modelagem computa- 
cional. Revista Brasileira de Ensino de Ciência e Tecnologia, v. 1, n. 1, p. 98115,2008

NORMAN, D. The invisible computer. Cambridge: MIT Press. 1998.

NRC (NATIONAL RESEARCH COUNCIL). National Science Education Standards. Washington, DC: The National Academic Press, 1996.

NSF (NATIONAL SCIENCE FOUNDATION). Setting a research and planning agenda for computer modelling in the pre college curriculum. Final Report. Grant RED-9255877, 1996.

OGBORN, J.; HOLLAND, D. Cellular modelling system. Microcomputer software. London: Longman. 1986.

OGBORN, J.; WONG, D. A Microcomputer dynamical modelling system. Physics Education, Bristol, UK, v. 19, p. 138-42, 1984.

OGBORN, J. Computational Modelling in Science. In: LEWIS, R.; TAGG, E. Trends in Computer Assisted Learning. Oxford: Blackwell, 1987. cap. 15. p. $148-66$.

OGBORN, J. Dynamic modelling system. Microcomputer software: system disc and physics models disc. London: Longman. 1984.

OGBORN, J.; BOOHAN, R.; WRIGHT, S. WorldMaker: a novel approach to computer modeling. User Guide, London: Institute of Education University of London, 1992.

OLIVEIRA, F. J. A construção de modelos baseada na utilização de diagramas causais no estudo de tópicos de ciências: um estudo com estudantes de Ensino Médio. 2004. 281 f. Dissertação (Mestrado em Física) - Centro de Ciências Exatas, UFES, Vitória, ES.

OLIVEIRA, R. R. A utilização da modelagem computacional qualitativa no estudo de difusão de gás: um estudo com estudantes universitários. 2006. 193 f. Dissertação (Mestrado em Física) - Centro de Ciências Exatas, UFES, Vitória, ES.

OLIVEIRA, R. R.; FERRACIOLI, L. O estudo do movimento retilíneo de três móveis a partir de uma atividade de modelagem computacional exploratória: uma 
aplicação no Ensino Médio a partir de módulos educacionais. In: ENCONTRO NACIONAL DE PESQUISA EM EDUCAÇÃO EM CIÊNCIAS, 8, 2011, Campinas, SP. Anais... Campinas, (no prelo) 2011.

OLIVEIRA, R. R.; FERRACIOLI, L. Variações do desempenho de alunos no estudo do movimento dos corpos: uma analise comparativa entre o desenvolvimento de atividades com modelagem computacional exploratória e atividades escolares tradicionais. In: ENCONTRO PESQUISA EM ENSINO DE FÍSICA, 14, 2012, Maresias, SP. Anais... Campinas, (no prelo) 2012.

PAPERT, S Mindstorm: children, computers and powerful ideas. New York: Basic Books, 1980. 215 p. [LOGO: Computadores e Educação. São Paulo: Brasiliense, 1985].

POLONINE, T.; GOMES, T.; FERRACIOLI, L. Módulo educacional sobre o cálculo de velocidade média em uma abordagem experimental utilizando o ambiente de modelagem computacional qualitativa ModeLab. In: SIMPÓSIO NACIONAL DE ENSINO DE FÍSICA, 18, 2009, Vitória, ES. 2009. Disponível em:

$<$ http://www.sbf1.sbfisica.org.br/eventos/snef/xviii/> Acesso em: 24 jan. 2012.

RABBI, M. A.; FERRACIOLI, L. O estudo de métodos numéricos de integração através da modelagem computacional quantitativa. In: ENCONTRO NACIONAL DE PESQUISA EM ENSINO DE FÍSICA, 8, 2002. Disponível em:

$<$ http://www.sbf1.sbfisica.org.br/eventos/epef/viii/>. Acesso em: 24 jan. 2012.

RAMPINELLI, M.; FERRACIOLI, L. A integração de um ambiente de modelagem computacional quantitativo no estudo do fenômeno de colisões. Caderno Brasileiro de Ensino de Física, Florianópolis, v. 23, n. 1, p. 93-122, 2006.

ROCHA, H. V.; BARANAUSKAS, M. C. Design e avaliação de interfaces humano-computador. Campinas: IC-NIED-UNICAMP. 2003.

ROGERS, E. M. Physics for inquiring minds: the method, nature and philosophy of Physical Science. Princeton, NJ: Princeton University Press, 1960.

SAMPAIO, F. F. WLinkIt: design, development and testing of a semiquantitative computer modelling tool. 1996. Tese (Doutorado em Educação) Institute of Education University of London, London, UK. 
VERBENO, C. A.; GOMES, T.; SILVA, R. M. A. da; FERRACIOLI, L. Investigação sobre a integração de um módulo educacional sobre o sistema mola-massa utilizando o ambiente de modelagem computacional SQRLab. In: SIMPÓSIO NACIONAL DE ENSINO DE FÍSICA, 19, 2011, Manaus, AM. Disponível em: $<$ http://www.sbfisica.org.br/ snef/xix/>. Acesso em: 24 jan. 2012

VICTOR, R. A.; FERRACIOLI, L. A utilização da modelagem computacional no laboratório de Física Básica. In: ENCONTRO NACIONAL DE PESQUISA EM ENSINO DE FÍSICA, 8, 2002. Disponível em:

$<$ http://www.sbf1.sbfisica.org.br/eventos/epef/viii/> Acesso em: 24 jan. 2012.

VITALE, B. Dix theses sur l'integration de l'informatique a la pratique pedagogique. In: COLLOQUE LOGO ET APPRENTISSAGE, 1991, Fribourg Suisse. [Publicado no Brasil: Computador na escola: um brinquedo a mais? Ciência Hoje, Rio de Janeiro, v. 13, n. 77, p. 18-25, mar. 1991.

VITALE, B. From local to global: programming and the unfolding of local models in the exploratory learning of Mathematics and Science. In: diSESSA, A.; HOYLES, C.; NOSS, R.; EDWARDS, L. Computers and exploratory learning. NATO ASI Series, v. 146. New York: Springer-Verlag, 1995.

VITALE, B. La integración de la informática en la aula: consideraciones generales para un enfoque transdisciplinar. Madrid: Visor Distribuidores, S.A. 1994.

VITALE, B. Representation and modelling of change over time by 12-13 year-old children in a scholl context. In: MONTANGERO, J.; WELLS, A.C.; TRYPHON, A.; VONÈCHE, J. Conceptions of change over time. Gèneve: Cahiers de la Foundation Archives J. Piaget, 1993. cap. 16. p. 279-290.

VITALE, B. The representation, the understanding and the mastering of experience: modelling and programming in a transdisciolinary context. Gèneva: Centre de Recherches Psycho-Pédagogique, 1995.

VITALE, B. Transforming familiar things and simple ideas into objects of knowledge. In: FERRACIOLI, L. CALDAS, H.; BISCH, S. M. Relatório final do projeto nordeste implantação da área de concentração Ensino de Física no Programa de Pós-Graduação em Física da Universidade Federal do Espírito Santo. Projeto Nordeste CAPES/COPLAG Convênio n. 03/97, Vitória, ES, 1998. 
WHITE, B.; HORWITZ, P. ThinkerTools: enabling children to understand physics laws. Final Report. Cambridge: BBN Report 6470. 1987. 